\title{
Improving Animal-Specific Radiotherapy Quality Assurance For Kilovoltage X-Ray Radiotherapy Using A 3D Printed Dog Skull Water Phantom
}

Yoshinori Tanabe ( $\nabla$ tanabey@yamaguchi-u.ac.jp )

Okayama University: Okayama Daigaku https://orcid.org/0000-0001-7259-3317

Toshie Iseri

Yamaguchi Daigaku

Ryouta Onizuka

Yamaguchi University Hospital: Yamaguchi Daigaku Igakubu Fuzoku Byoin

Takayuki Ishida

Osaka Daigaku

\section{Hidetoshi Eto}

Yamaguchi University Hospital: Yamaguchi Daigaku Igakubu Fuzoku Byoin

Munekazu Nakaichi

Yamaguchi Daigaku

\section{Research Article}

Keywords: orthovoltage, animal radiotherapy, heel effect, water animal phantom, animal specific radiotherapy quality assurance

Posted Date: September 7th, 2021

DOl: https://doi.org/10.21203/rs.3.rs-844438/v1

License: (c) (i) This work is licensed under a Creative Commons Attribution 4.0 International License. Read Full License 


\section{Abstract}

Accurate dose assessment during animal radiotherapy is beneficial for veterinary medicine and medical education. We evaluated the dose distributions of kilovoltage $X$-ray orthovoltage radiotherapy and created a dog skull water phantom for animal-specific radiotherapy. EGSnrc-based BEAMnrc and DOSXYZnrc codes were used to simulate orthovoltage dose distributions. At 10, 20, 30, 40, 50 and $80 \mathrm{~mm}$ in a water phantom, depth dose was measured with waterproof Farmer dosimetry chambers and the diagonal offaxis ratio was measured with Gafchromic EBT3 film to simulate orthovoltage dose distributions. Energy differences between orthovoltage and linear accelerated radiotherapy were assessed with a heterogeneous bone and tissue virtual phantom. The animal-specific phantom for radiotherapy quality assurance was created from CT scans of a dog and printed with a three-dimensional printer using polyamide 12 nylon, with insertion points for dosimetry chambers and Gafchromic EBT3 film. Monte Carlo simulated and measured dose distributions differed by no more than $2.0 \%$ along the central axis up to a depth of $80 \mathrm{~mm}$. The anode heel effect occurred in shallow areas. The orthovoltage radiotherapy percentage depth dose in bone was $>40 \%$. Build-up was $>40 \%$, with build-down after bone exit, whereas linear accelerator radiotherapy absorption changed little in the bone. A highly water-impermeable, animalspecific dog skull water phantom could be created to evaluate dose distribution.Animal-specific water phantoms and Monte Carlo simulated pre-treatment radiotherapy is useful quality assurance for

orthovoltage radiotherapy and yields a visually familiar phantom that will be useful for veterinary medical education.

\section{Introduction}

Small animal radiotherapy requires high treatment accuracy and animal-specific radiotherapy quality assurance (QA). Dose accuracy in animal models has been studied as a way to estimate the radiation response of tumors and normal tissues [1].

Kilovoltage X-rays delivered via orthovoltage equipment (Philips Medical Systems, Best Netherlands) for small animal radiotherapy are widely used in facilities ranging from clinics to university hospitals [2,3]. The use of linear accelerators for animal radiotherapy has increased in some facilities, but remains rare due to high costs and large machine size [2]. The absorbed dose during orthovoltage radiotherapy may be measured using water procedures [4] to ensure that the prescribed doses for soft tissue are accurate. However, differences in tissue density throughout the body (e.g. lung and bone) make it difficult to accurately predict dose distribution in clinical use. Equipment and patient-specific QA are important for identifying discrepancies between calculated and delivered radiation doses and for assessing algorithmbased management of heterogeneities and other factors $[5,6]$. Orthovoltage radiotherapy is delivered in high prescription doses, similar to linear accelerator radiotherapy $[3,7]$, but manufacturers generally do not sell radiation treatment planning systems.

Monte Carlo simulation is an established method for estimating radiation treatment dose [8, 9], including for orthovoltage radiotherapy dose distribution [10]. If the orthovoltage dose distribution can be 
understood, this technique may be an effective tool for selecting either orthovoltage or linear accelerator radiotherapy when considering dosing around radiosensitive organs. Yet, in veterinary education there is little time to teach about radiation therapy, and the use of visual simulation images and animal-specific verification devices can aid in our understanding of the physical events of radiation in a shortened time [11]

Three-dimensional (3D) printers are widely used in radiotherapy research to create phantoms from processed CT images $[12,13]$. The accuracy of 3D printers depends on the printing materials and image processing methods. The accuracy required for QA verification is within $1 \mathrm{~mm}$ [5], and engineering technology and knowledge are essential for creating high-quality phantoms.

To the best of our knowledge, there are no prior reports on the use of a 3D printed dog skull water phantom for pre-treatment QA of orthovoltage radiotherapy dose. We investigated the ability of Monte Carlo simulations and physical phantom measurements to predict dose distributions with conventional orthovoltage X-ray equipment and enable the creation of a dog skull water phantom that could be used for QA. Additionally, the suitability of orthovoltage radiotherapy was compared with accelerated linear radiotherapy.

\section{Methods}

This study used a self-contained industrial orthovoltage X-ray system (output voltage rage 32-320 kV, tube current range 3-30 mA; Philips Medical Systems, Best, Netherlands) that is generally used for small animal radiotherapy. The unit has several applicator cones, a field size of $\varphi 4.0 \mathrm{~cm}$ and a treatment distance of $50 \mathrm{~cm}$.

\section{Monte Carlo simulation for orthovoltage radiotherapy}

Monte Carlo orthovoltage dose distributions were simulated with EGSnrc-based BEAMnrc and DOSXYZnrc codes using MATLAB from the NRCC group [14, 15]. The orthovoltage apparatus consists of a target, X-ray tube window, primary collimator, and mirror. The geometric dimensions and materials of each component module were provided by vendors and obtained from reference tables $[16,17]$. The incident electron energy was set to $300 \mathrm{kV}$, with an added filtration of $2.0 \mathrm{~mm}$ aluminum and $0.5 \mathrm{~mm}$ copper. The percentage dose depth was measured at 10, 20, 30, 40, 50 and $80 \mathrm{~mm}$ with standard PTW 300130.6 cc waterproof Farmer ionization chambers (PTW, Freiburg, Germany) in a WP1D water phantom (IBA Dosimetry, Schwarzenbruck, Germany), and these physical measurements in the water phantom were used as comparators for the simulated doses. The diagonal off-axis ratio for each depth was measured with the Gafchromic EBT3 film (Ashland Advanced Materials, Bridgewater, NJ, USA) and a tough water phantom (Kyoto Kagaku, Kyoto, Japan) to model the distribution spread for the Monte Carlo simulation. 
The dose distributions in the model dog CT image were compared to those in the Monte Carlo simulations for this study's orthovoltage radiotherapy and those for an Agility MV voltage linear accelerator (Elekta AB, Stockholm, Sweden) using previously published models [9].

We assessed the effects of energy differences on bone by creating a heterogeneous bone and tissue phantom consisting of a $1.0 \mathrm{~cm}$ thick layer of water above a $1.0 \mathrm{~cm}$ thick layer of cortical bone (physical density $\rho=1.40 \mathrm{~g} / \mathrm{cm}^{3}$ ) and a $10 \mathrm{~cm}$ layer of water. Dose profiles were compared between orthovoltage radiotherapy (energy $300 \mathrm{KeV}$ and a field size of $4.0 \times 4.0 \mathrm{~cm}^{2}$ ) and the Agility MV voltage linear accelerator (6 MV and a field size of $\left.4.0 \times 4.0 \mathrm{~cm}^{2}\right)$.

\section{Printing of the dog skull water phantom}

The 3D printed dog skull water phantom was modeled on a medium-sized Shiba Inu $(\sim 12 \mathrm{~kg})$ suitable for treatment with orthovoltage or linear accelerator radiotherapy. Using a 3D workstation (Ziostation-2; Ziosoft/AMIN, Tokyo, Japan), 3D CT raw images of the dog were processed for erosion. Structures other than the $3 \mathrm{~mm}$ surface were removed from the image after subtraction processing, and a STL image of only the $3 \mathrm{~mm}$ thick surface was created. Next, using the CAD STL data editing software GeoMagic Design X (3D Systems, Rock Hill, SC USA) and SOLIDWORKS 3D CAD Premium 2020 (Dassault Systèmes, Vélizy-Villacoublay, France), a dog-shaped water phantom was designed that included insertion points for the Farmer chamber dosimeters and two Gafchromic films. A RaFaEl II plus 300C-HT 3E printer (Aspect Co., Tokyo, Japan) was used to create the phantom with polyamide 12 nylon [18] in order to make it impermeable to water, and an acrylic paint was applied to the surface to suppress gas permeability (Table 1$)$.

Table 1 Material and geometry information for a dog skull water phantom

Dog skull water phantom

3D printing

Material

Specific gravity

Particle size $(\mu \mathrm{m})$

Joining

Surface coating

Distance from the center of the Farmer chamber to the surface $(\mathrm{mm})$

Distance from Gafchromic film (1st) to surface ( $\mathrm{mm}$ )

Distance from Gafchromic film (1st) to nose surface (mm)
Additive manufacturing techniques

Polyamide 12 nylon

1.03

$45 \pm 5$

Cemedine PPX

Acrylic paint

16.0

33.2

15.0 


\section{Results}

Figure 2 shows the radiation dose profile along the axis, measured at depths of $5,10,15,20,30,40,50$ and $80 \mathrm{~mm}$. Table 2 shows the dose ratio difference up to $10 \mathrm{~mm}$ off axis on each side (the anode heel effect). The in-plane dose along the profile was reduced by $1 \%$ up to $15 \mathrm{~mm}$ on the anode side. The irradiation diameter obtained at a dose of $50 \%$ at a depth of $2 \mathrm{~cm}$ was asymmetric (in plane $3.64 \mathrm{~cm}$; cross plane $4.26 \mathrm{~cm}$ ).

Table 2 The ratio of dose difference for $10 \mathrm{~mm}$ off axis at each side

\begin{tabular}{|lllllllll|}
\hline depth $(\mathrm{mm})$ & 5 & 10 & 15 & 20 & 30 & 40 & 50 & 80 \\
\hline $\begin{array}{l}\text { In plane } \\
(\%)\end{array}$ & -1.78 & -1.26 & -1.49 & -0.96 & -0.55 & 0.22 & -0.32 & -0.33 \\
$\begin{array}{l}\text { Cross plane } \\
(\%)\end{array}$ & 0.51 & 0.74 & 0.74 & 0.42 & -0.11 & -0.10 & 0.00 & -0.21 \\
\hline
\end{tabular}

Figure 3 shows the Monte Carlo simulation percentage depth dose and the measured percentage depth dose. The dose difference between the measured dose and beam modeling was adjusted to within at least $2.0 \%$ along the central axis.

Figure 4 shows the radiation dose distributions estimated by Monte Carlo simulations for linear accelerator radiotherapy under near-irradiation conditions and for orthovoltage radiation. The absorbed dose to the skull near the surface with orthovoltage radiation was similar to that of the single port irradiation, and the reduction rate of orthovoltage radiation was high.

The percentage depth dose in bone with orthovoltage radiotherapy was more than $40 \%$. With orthovoltage radiotherapy a build-up of more than $40 \%$ was seen in the bone region, with build-down after bone transmission (Fig. 5a). With linear accelerator radiotherapy there was little change in absorption in the bone region, although a slight rebound was seen after bone transmission (Fig. 5b).

Figure 6 shows the water phantom that was created using a 3D printer. The dog skull water phantom was water impermeable, had inserts for Farmer dosimetry chambers and two sheets of Gafchromic film, and could be used to perform animal-specific radiotherapy QA.

\section{Discussion}

In small animals, radiotherapy is often performed in shallow areas. Use of Monte Carlo simulations to aid dosimetry is useful for evaluating dose distribution, but in veterinary medicine education visual simulation phantoms can be quicker to use and learn from. In this study the use of simulated doses and measurements in a water phantom provided us with an understanding that could be applied to a create a 3D printed animal-specific dog skull water phantom with appropriate inserts for dosimetry chambers and 
Gafchromic sheets, that was water impermeable and had high gas impermeability, and permitted pretreatment radiotherapy QA.

As shown by the results of the off-axis ratios, the heel effect is seen at a shallow depth of about $1.5 \mathrm{~cm}$, a finding that is reflected in past research $[17,19]$. The actual radiation field size was asymmetric, which may change over time because this study's orthovoltage machine was made more than 20 years after its introduction. Additionally, since vendors fine-tune the beam quality and target angle at each facility, we believe that beam modeling per facility, as for linear accelerator equipment, is important.

Based on the comparison of orthovoltage and linear accelerator radiotherapy, the absorbed dose was similar to that of the linear accelerator in bone near the surface, but the orthovoltage radiation had greater attenuation as bone depth increased. The interaction between photons and matter is greatly affected by the energy range. The photoelectric effect region for orthovoltage radiation is affected by atomic number, in contrast to the Compton scattering region, which affects linear accelerator radiation [20]. We believe that the visualization of the dose distribution accuracy of orthovoltage radiotherapy for veterinary medicine is useful for evaluating targets and at-risk organs. Furthermore, the dose distribution serves as a criterion for selecting either orthovoltage or linear accelerator radiotherapy.

The major limitation to this study is that since the measurement position of the animal phantom is fixed, its degrees of freedom are lower than those of a solid water phantom. It is therefore necessary to understand the merits of both. However, education on radiation therapy in veterinary medicine is limited. The water dog phantom makes it possible to understand the differences in dose between the orthovoltage and linear accelerator radiotherapy with a familiarly shaped animal phantom rather than a rectangular phantom. We therefore believe that we can help improve and reduce the time needed for radiotherapy veterinary education with our method for creating animal-specific phantoms.

In this study a 3D printer was able to create an accurate water impermeable phantom by means of the knowledge and technical cooperation of the engineering department. Dose evaluation can be performed using medical experience and knowledge, illustrating that cooperation between veterinary medicine, medicine and engineering is effective.

\section{Conclusions}

By using Monte Carlo simulations, physical phantom measurements and a 3D printer, we were able to visualize the radiation treatment distribution of orthovoltage X-ray equipment in clinical practice. This techniquye will be beneficial for animal-specific radiotherapy QA and veterinary medicine education.

\section{Declarations}

Funding: We gratefully acknowledge support from the Japanese Society of Radiological Technology (JSRT) Research Grant (2018). 
Conflicts of interest/Competing interests: The authors declare no conflicts of interest.

Non-financial interests: None

Availability of data and material: Not applicable

Code availability: Not applicable

Authors' contributions: Not applicable

Ethics approval: None

Consent to participate: The IRB waived the need to obtain patient consent owing to the retrospective nature of the study.

Consent for publication: Not applicable

\section{References}

1. Verhaegen F, Dubois L, Gianolini S et al (2018) ESTRO ACROP: technology for precision small animal radiotherapy research: optimal use and challenges. Radiother Oncol 126:471-478. doi:https://doi.org/10.1016/j.radonc.2017.11.016

2. Seo J, Son J, Cho Y et al (2018) Kilovoltage radiotherapy for companion animals: dosimetric comparison of $300 \mathrm{kV}, 450 \mathrm{kV}$, and $6 \mathrm{MV}$ X-ray beams. J Vet Sci 19(4):550-556. https://doi.org/10.4142/jvs.2018.19.4.550

3. Pampena R, Palmieri T, Kyrgidis A et al (2016) Orthovoltage radiotherapy for nonmelanoma skin cancer (NMSC): comparison between 2 different schedules. J Am Acad Dermatol 74(2):341-347

4. Ma CM, Coffey CW, DeWerd LA et al (2001) AAPM protocol for 40-300 kV x-ray beam dosimetry in radiotherapy and radiobiology. Med Phys 28:868-893

5. Miften M, Olch A, Mihailidis D et al (2018) Tolerance limits and methodologies for IMRT measurement-based verification QA: Recommendations of AAPM Task Group No. 218. Med Phys 45:e53-e83. doi:10.1002/mp.12810

6. Tanabe Y, Ishida T, Eto H, Sera T, Emoto Y, Shimokawa M (2020) Patient-specific radiotherapy quality assurance for estimating actual treatment dose. Med Dosim. doi:10.1016/j.meddos.2020.08.003

7. Medina LA, Herrera-Penilla BI, Castro-Morales MA et al (2008) Use of an orthovoltage X-ray treatment unit as a radiation research system in a small animal cancer model. J Exp Clin Cancer Res 27:57

8. Andreo P (1991) Monte Carlo techniques in Medical Radiation Physics. Phys Med Biol 36:861-920

9. Onizuka R, Araki F, Ohno T. Monte carlo dose verification of VMAT treatment plans using elekta agility 160-leaf MLC. Physica Med. 2018;22-31

10. Abbas H, Mahato DN, Satti J, MacDonald CA (2014) Measurements and simulations of focused beam for orthovoltage therapy Med. Phys 41:041702 
11. Azer SA, Azer S (2016) 3D anatomy models and impact on learning: a review of the quality of the literature. Heal Prof Educ 2:80-98. doi:10.1016/j.hpe.2016.05.002

12. Ji Z, Jiang Y, Guo F et al (2017) Dosimetry verification of radioactive seed implantation for malignant tumors assisted by 3D printing individual templates and CT guidance. Appl Radiat Isot 124:68-74

13. Kamomae T, Shimizu H, Nakaya T et al (2017) Three-dimensional printer-generated patient-specific phantom for artificial in vivo dosimetry in radiotherapy quality assurance. Phys Med 44:205-211

14. Kawrakow I (2000) Accurate condensed history Monte Carlo simulation of electron transport. I. EGSnrc, the new EGS4 version. Med Phys 27:485-498

15. Kawrakow I, Rogers DWO, Tessier F et al (2011) The EGSnrc code system: Monte Carlo simulation of electron and photon transport. National Research Council of Canada Report PIRS-701

16. Abbas H, Mahato DN, Satti J, MacDonald CA (2014) Measurements and simulations of focused beam for orthovoltage therapy. Med Phys 41:041702

17. Knoos T, Rosenschold PMa, Wieslander E. Modelling of an orthovoltage x-ray therapy unit with the EGSnrc Monte Carlo package. J Phys: Conf Ser. 2007;74:021009. doi:10.1088/17426596/74/1/021009

18. Rahim TNAT, Abdullah AM, Akil HM, Mohamad D. Comparison of mechanical properties for polyamide 12 composite-based biomaterials fabricated by fused filament fabrication and injection molding. In AIP Conference Proceedings, 1791, 2016

19. Azim R, Alaei P, Spezi E, Hui SK (2015) Characterization of an orthovoltage biological irradiator used for radiobiological research. J Radiat Res 56:485-492

20. Jones KC, Redler G, Templeton A, Bernard D, Turian J, Chu JCH (2018) Characterization of Comptonscatter imaging with an analytical simulation method. Phys Med Biol 63:025016

\section{Figures}

a
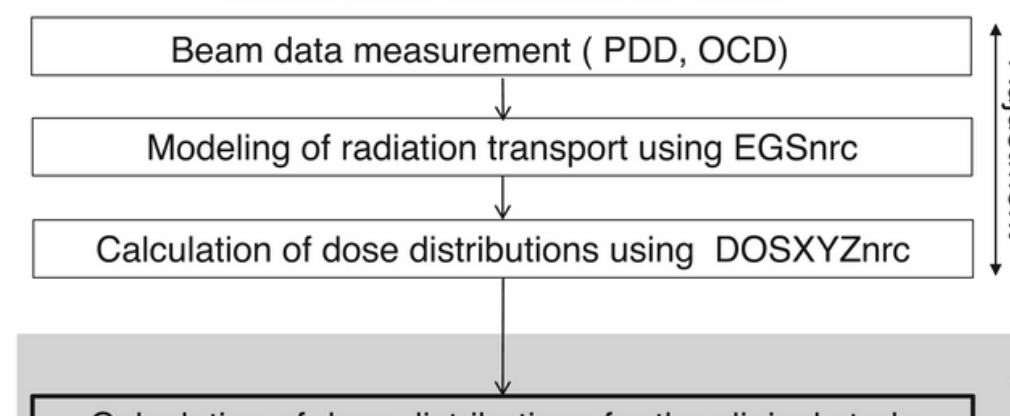

Calculation of dose distributions for the clinical study b Measurement system

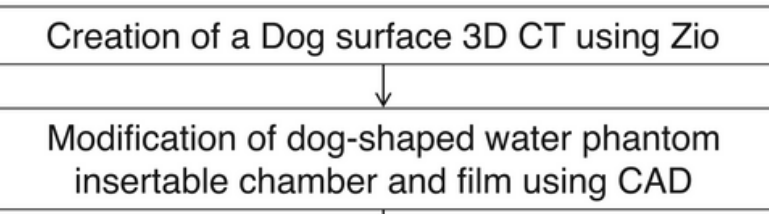

Creation of a dog-shaped water phantom using a 3D printer

\section{The animal-specific radiotherapy quality assurance}

\section{Figure 1}


Flowchart of the current study. (a: Monte Carlo simulation for orthovoltage radiotherapy using EGSnrcbased BEAMnrc and DOSXYZnrc codes; b: Measurement system using 3D printed dog skull water phantom)
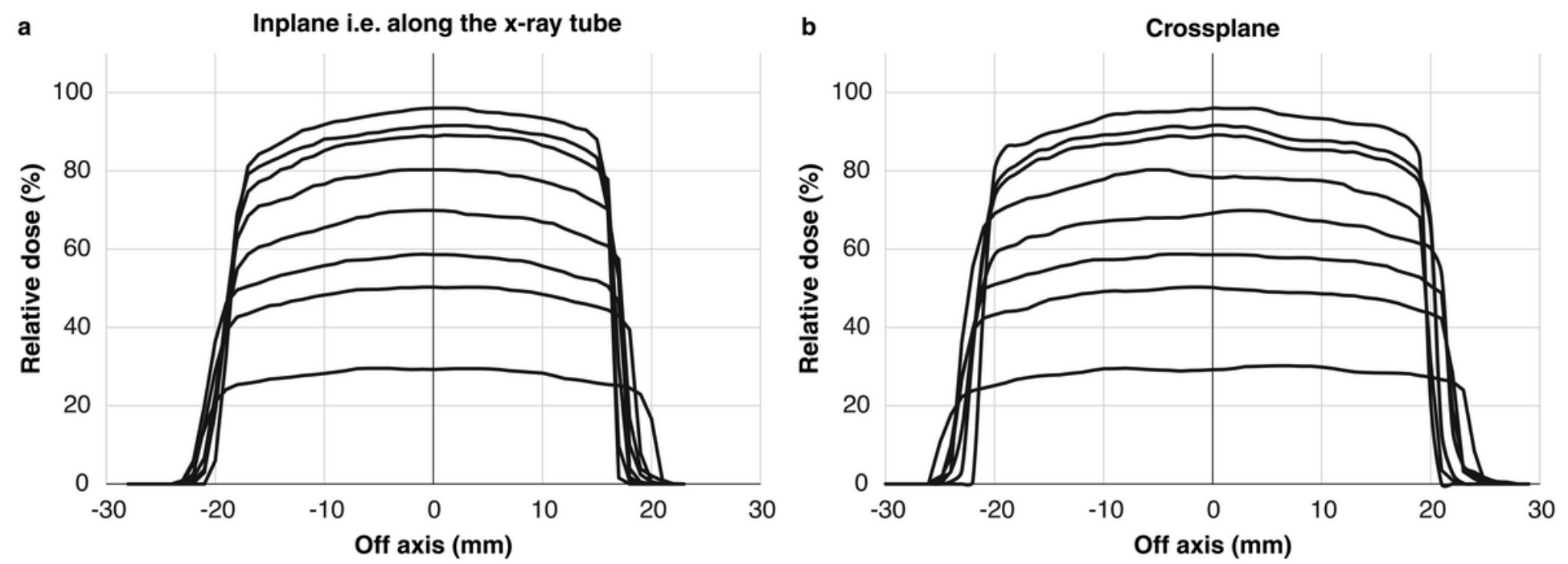

Figure 2

Measured dose profiles along the two axes using film (a: in plane i.e. along the X-ray tube; b: cross plane). 


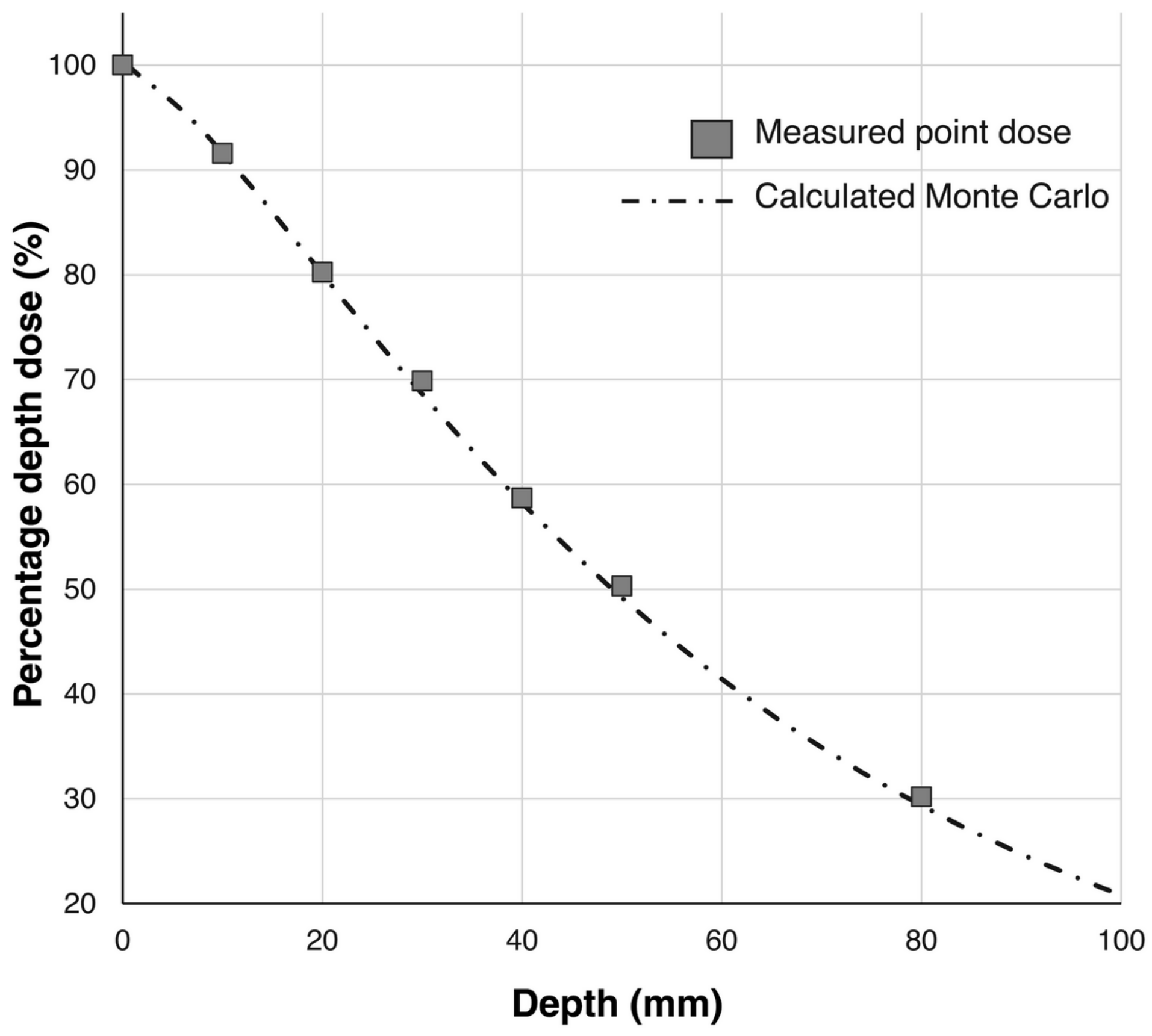

Figure 3

Depth dose curves for orthovoltage radiotherapy. The graph compares a Monte Carlo simulation and water measurements. 
a

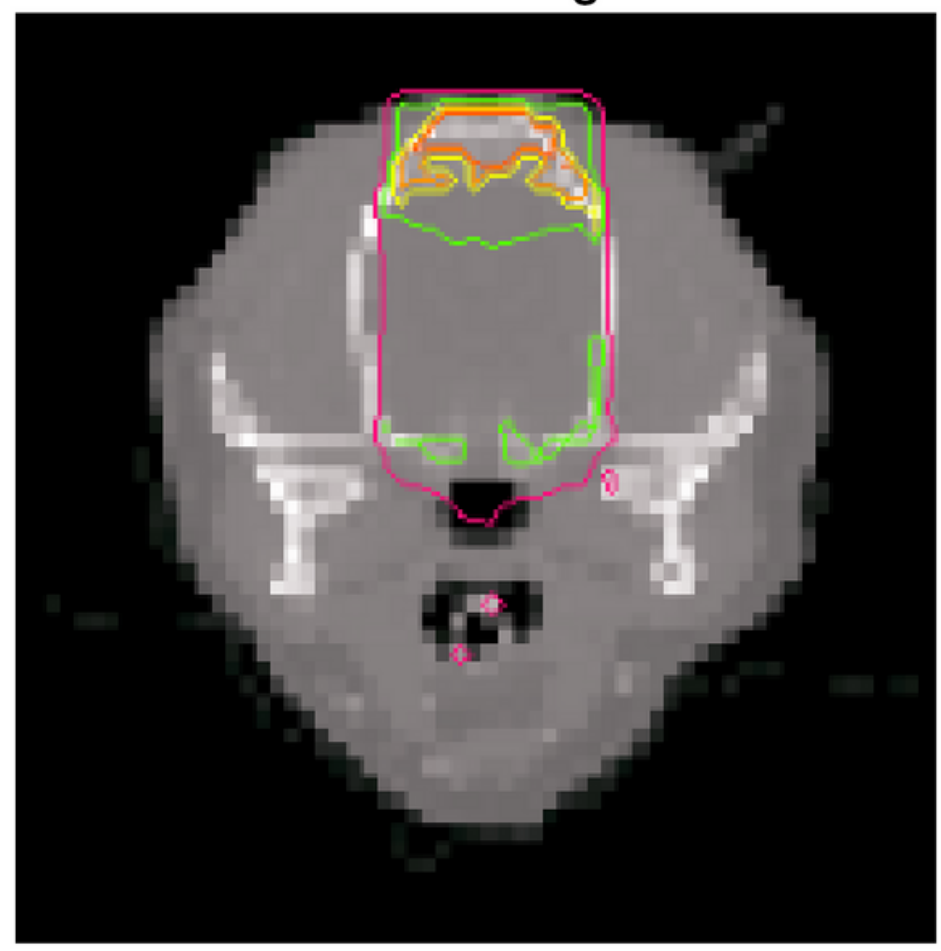

b

Linear accelerator

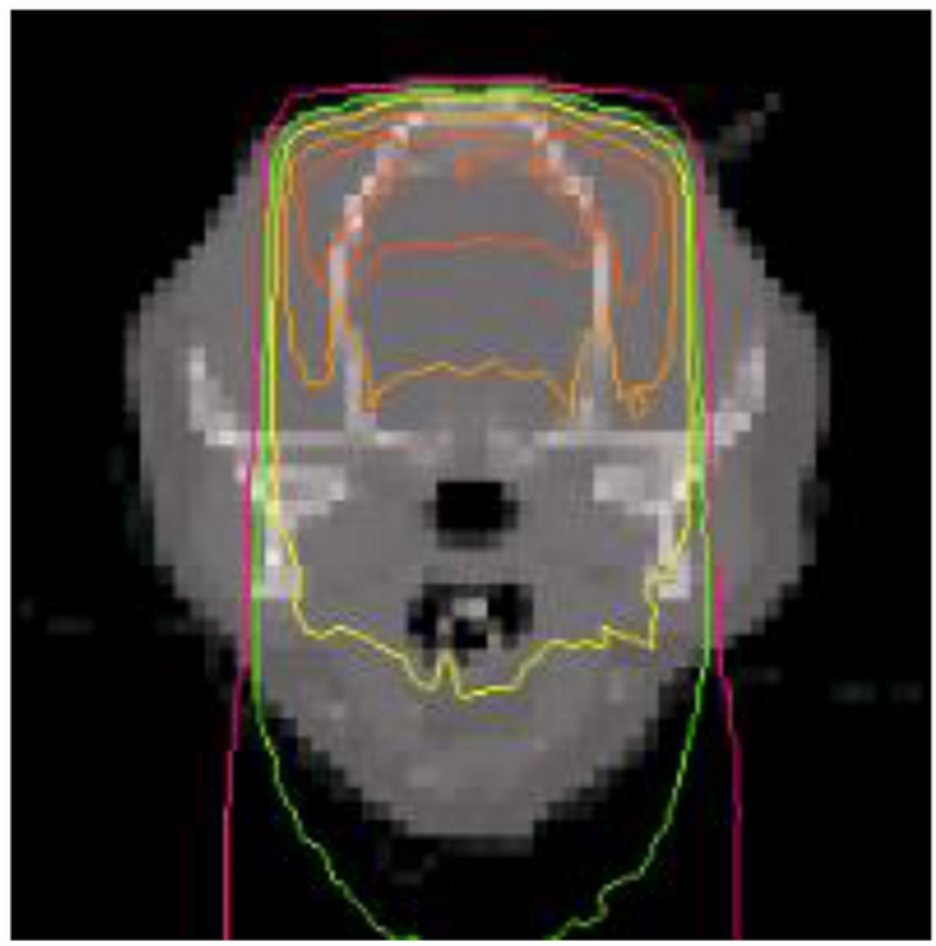

Figure 4

Dose distribution using Monte Carlo simulations. (a: orthovoltage radiotherapy; b: linear accelerator radiotherapy)
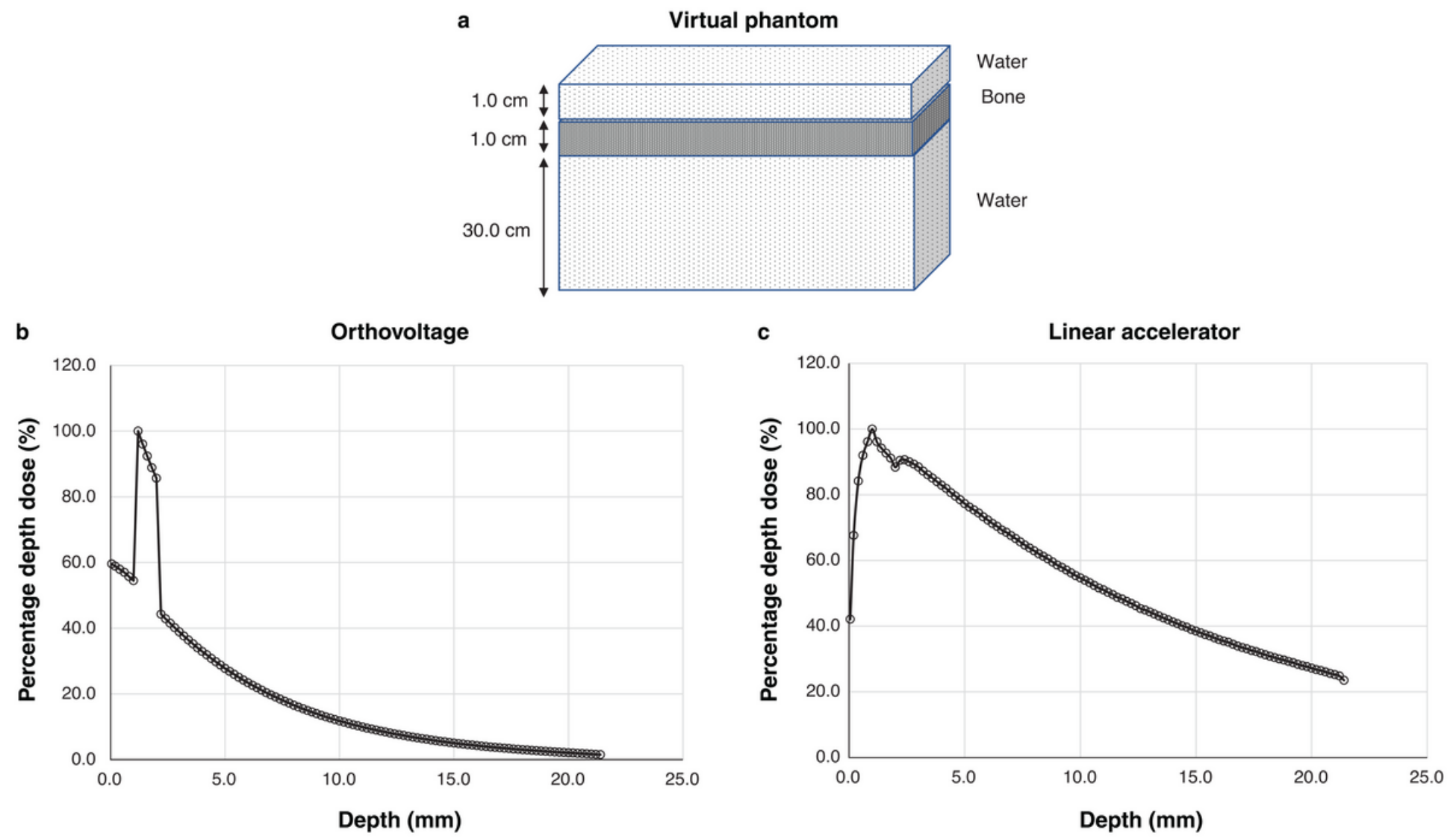
Figure 5

Dose curve for the heterogeneous bone and tissue phantom (a: virtual phantom setting; $b$ : the dose curve with orthovoltage radiotherapy; c: the dose curve of the linear accelerator radiotherapy)
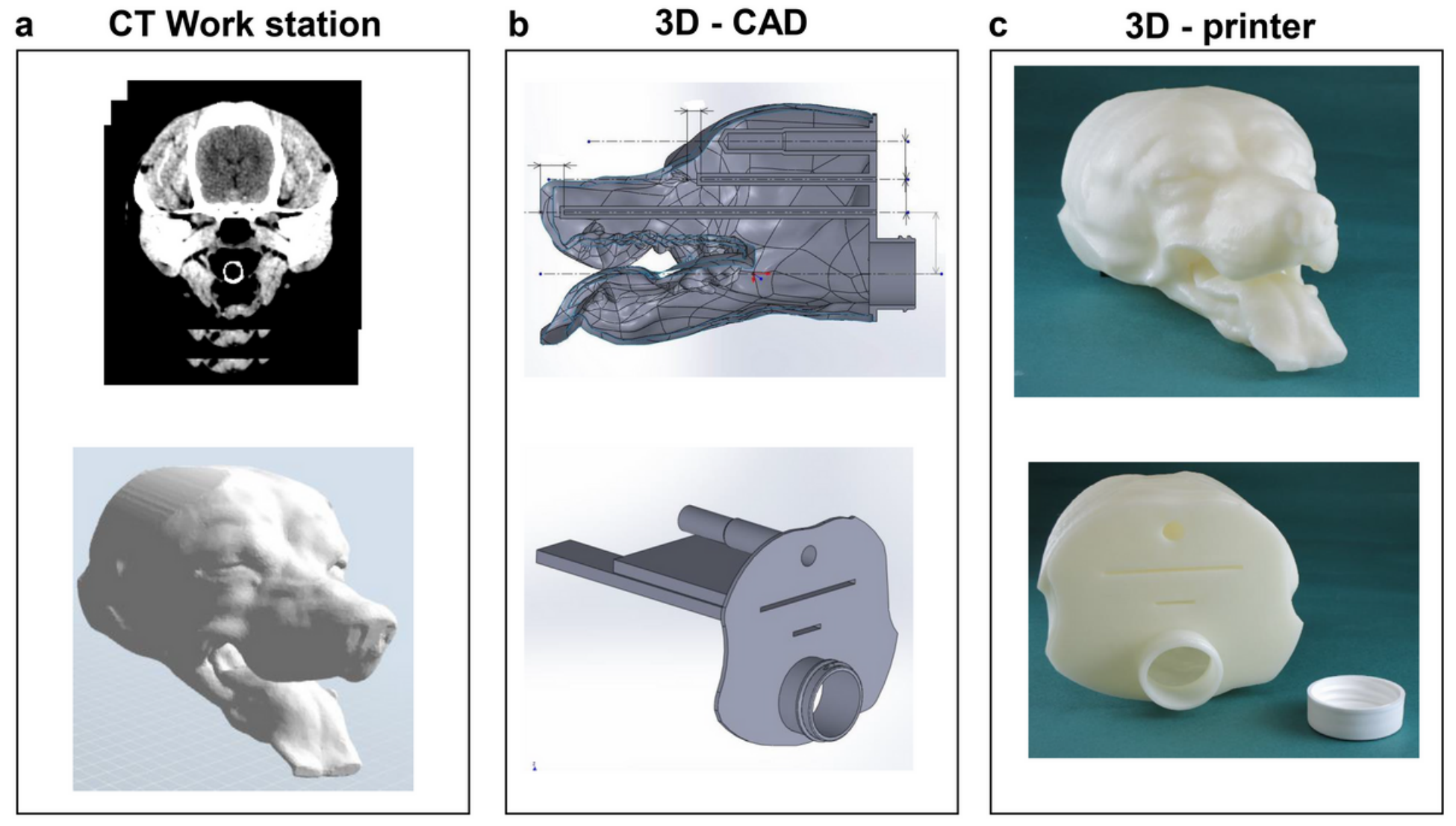

\section{Figure 6}

Workflow for creating the dog skull water phantom. (a: 3D workstation; b: 3D-CAD; c: 3D printer) 